\title{
A Novel System on Efficient Matching, Decision Making and Distributing
}

\author{
Si-Yuan Liü,2, Yu Liư ${ }^{3}$, Chao Liư ${ }^{4}$ Chun-Zhe Zhao ${ }^{5}$, Yi-Ming Luo ${ }^{2}$, Gao-Jin Wen ${ }^{1}$
}

${ }^{1}$ Shenzhen Institute of Advanced Technology, Chinese Academy of Sciences, Shenzhen, China; ${ }^{2}$ The Hong Kong University of Science and Technology, Hongkong, China; ${ }^{3} \mathrm{KTH}$ Royal Institute of Technology, Stockholm, Sweden; ${ }^{4}$ Institute of Computing Technology, Chinese Academy of Sciences, Beijing, China; ${ }^{5}$ Academy of Mathematics and System Science, Chinese Academy of Sciences, Beijing, China.

Email: syliu@ust.hk

Received 14 April 2009; revised 2 May 2009; accepted 4 May 2009.

\begin{abstract}
The object matching and distribution problem is a traditional challenge in different kinds of networks, such as kidney distribution networks. Applying differential element analysis methods, decision tree, integer linear programming theory and stochastic processes ideas, we propose models for the objects matching, the distribution network, the exchange system and the individual decision-making strategy, and thoroughly analyze the relationship between the matching rate and the waiting time, and their impacts on the efficiency of the donor-matching process. And as the experiments, we evaluate the algorithms and system by kidney matching, decision making and distribution problems on real world data.
\end{abstract}

Keywords: biological system modeling; computer aided analysis; computer aided software engineering; decision-making

\section{INTRODUCTION}

The matching and distribution problem is not only a problem in mathematics, and computer science, but also in medical service. In this paper, we take kidney matching and exchanging as a study case to try to shed light into the matching and distribution problem.

Despite the advances in medicine and health technology, the demand for organs for transplantation drastically exceeds the number of donors. Organs for transplant are obtained either from a cadaver queue or from living donors. The keys for the effective use of the cadaver queue are cooperation and good communication throughout the network. But unfortunately, the network needs a lot of improvements to realize an efficient donor-matching process, save and prolong more lives and provide strategies for the patients to get a better kidney.

Based on mathematical models, IT, Ethnics, Sociol- ogy, Medicine, laws, policies, criteria and so on, we design a scalable service oriented architecture (SOA) system and network, which will improve the efficiency of the donor-matching process, the kidney distribution process, evaluate strategies for the patients to make the decision, and save and prolong more lives [16].

In this paper, we address and emphases mathematical models for better kidney matching, distribution, exchanges, and personal strategies.

\subsection{Scalable SOA Network System}

To make ways to share information on kidney donor, matching and transplant much faster, fairer and public, we should establish a national scalable service-oriented architecture (SOA) system and network (the development process refers to Figure 1, and the architecture refers to Figure 2) to collect, organize and put out the information [11].

The aspects of the service definition in SOA:

1. Services are defined by explicit, implementationindependent interfaces.

2. Services are loosely bound and invoked through communication protocols that stress location transparency and interoperability.

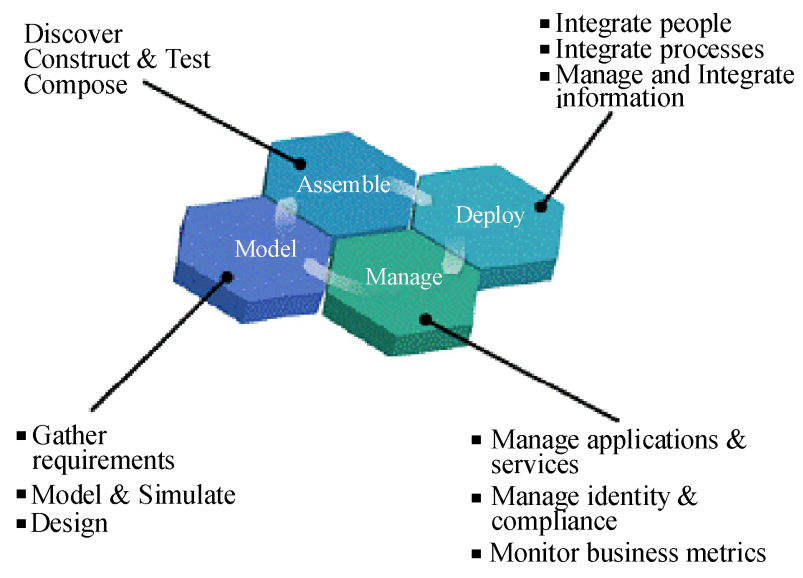

Figure 1. Development process [17,18]. 
3. Services encapsulate reusable business function.

4. Services to good communication and more donors for patients.

The service flow is as Figure $\mathbf{3}$ shows.

The system and network should also be designed to base on web service, which provide an emerging set of open-standard technologies that can be combined with proven existing technologies to implement the concepts and techniques of SOA.

Based on this kind of scheme, the transplant network will come to a better status, that is, a better communication (which has been processed as services), a better efficiency, a better robustness and a better scalability [11], especially making the communication and information sharing aggregated services in a distributed heterogeneous network computing environment.

\section{MATHEMATICAL MODELS FOR THE TRANSPLANT NETWORK}

\subsection{Analysis of the Problem}

The popular method nowadays is, to collect personal information, evaluate the patients' situation and give a score, and then sort the patients by the score. When a kidney appears and is available, the patient who has a better matching rate, a higher priority and a higher score will get it. This kind of scheme may come to an approximate best result, but after our extensive research, a rigorous reasoning and innovative designs, we propose a new program which will should save and prolong lives as more as possible, and meanwhile cut the waiting time for the patient as short as possible.

\subsection{Assumptions}

1) Everybody' life obeys exponential distribution.

2) Malthus Model, Logistic Model and Regressive Model are applied to forecast the population changing in a short term (a year) [3].

3) The population can be divided into four classes:

a) Normal persons live without nephropathy, and with two kidneys.

b) Normal persons live with only one kidney because of a living donor.

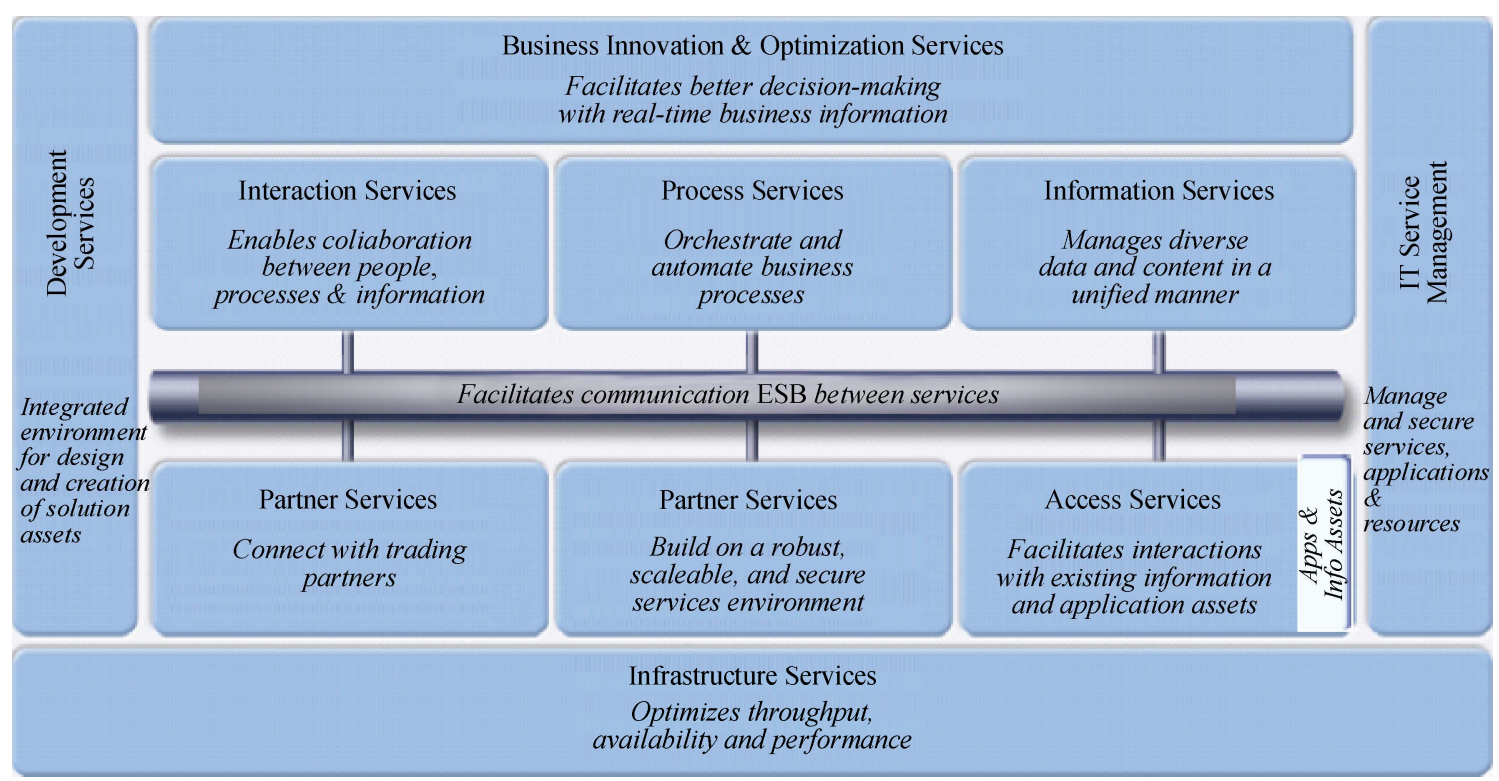

Figure 2. Service oriented architecture $[16,17,18]$.
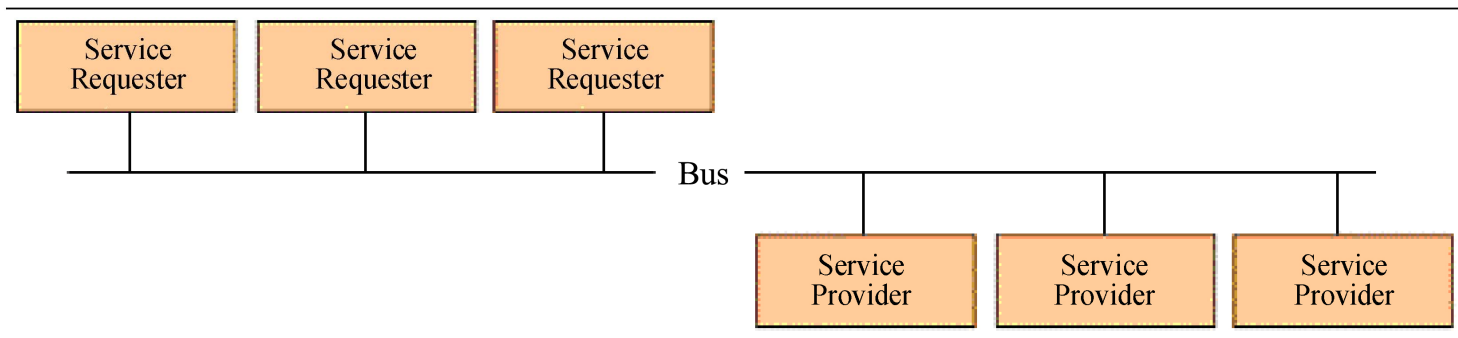

Figure 3. SOA service flow [17]. 
c) The patients wait for cadaver and living donors, including renal allograft dysfunctions after the first transplant.

d) The patients are healthy now after transplant.

4) Because the demand for organs transplantation drastically exceeds the number of donors, we assume, physiologically, any kidney can get a match patient.

5) The transplant kidney's life obeys exponential distribution.

6) The death rate of the patient in the waiting list is higher than that of the population, but after transplant operation, before the renal allograft dysfunctions occur, the death rate of the patient is considered to be the same as the normal, and from the study material, the death rate of the operation is very low, maybe can be considered to be 0 .

\subsection{Definitions and Key Terms}

$t$ is time, unit: year.

$x_{1}$ is the percentage of the patients waiting for donors in the total population.

$x_{2}$ is the percentage of healthy patients after transplant in the total population.

$x_{3}$ is the percentage of available cadavers in the total population at $t$.

$x_{4}$ is the percentage of healthy patients after living donors transplant in the total population at $t$.

$N(t)$ is the total population at $t$.

$X_{i}(t)=x_{i} N(t), \quad i=1,2,3,4$.

a is the probability of serious kidney disease which the patients need transplant in the population in a time unit.

$\mathrm{b}$ is the death rate of the patients in the waiting list.

$\mathrm{c}$ is the rate of renal allograft dysfunction.

$\mathrm{d}$ is the average death rate in the total population.

$\mathrm{h}$ is the average number of kidneys from living donors for a patient.

$\mathrm{k}$ is the productivity of the available cadavers in the total population.

\subsection{Design of the Model}

The variation of $X_{1}(t)$ at $[t, t+\Delta t]$ is $X(t+\Delta t)-$ $X(t)$ which should include the new increasing patients, $a\left(N-X_{1}-X_{2}\right) \Delta t$, from $t$ to $t+\Delta t$, the number of patients, $c x_{2} \Delta t$, who have to wait for donors again because of renal allograft dysfunction and the variation, $-b x_{1} \Delta t-2 x_{3} \Delta t-h x_{1} \Delta t$, because of death, cadavers and living donors.

So,

$$
\begin{aligned}
& X_{1}(t+D t)-X_{1}(t)= \\
& a\left(N-X_{1}-X_{2}\right) D t+c X_{2} D t-b x_{1} D t-2 x_{3} D t-h x_{1} D t
\end{aligned}
$$

$$
\frac{X_{1}(t+\Delta t)-X_{1}(t)}{\Delta t}=(a-b-h) X_{1}+(c-a) X_{2}-2 X_{3}+a N
$$

Let $\Delta t \rightarrow 0$, so

$$
\dot{X}_{1}(t)=(a-b-h) X_{1}+(c-a) X_{2}-2 X_{3}+a N
$$

while the variation of $X_{2}$ mainly depends on renal allograft dysfunction, the decrease from the normal death and the increase from cadaver and living donors, so

$$
X_{2}(t+\Delta t)-X_{2}(t)=\left[h X_{1}+(-c-d) X_{2}+2 X_{3}\right] \Delta t
$$

And then,

$$
\dot{X}_{2}(t)=h X_{1}+(-c-d) X_{2}+2 X_{3}
$$

The variation of available cadavers, $X_{3}$, is a direct ratio with the number of the healthy people at $t$, but, the people who has made a living donor will only donate a kidney after death, so

$$
\begin{aligned}
& X_{3}(t+\Delta t)-X_{3}(t)=k\left(N-X_{1}-X_{4}-X_{3}\right) \Delta t+\frac{1}{2} k X_{4} \Delta t= \\
& k\left(N-X_{1}-X_{3}-\frac{1}{2} X_{4}\right) \Delta t
\end{aligned}
$$

And then,

$$
\dot{X}_{3}(t)=-k X_{1}-k X_{2}-\frac{1}{2} k X_{4}+k N \text {. }
$$

Last but not least, we analyze the variation of $X_{4}$.

Based on common sense, we would come to an analysis that the living donor always chooses a direct transplant to relatives or friends, so the variation of $X_{4}$ is strongly relative to the variation of $X_{1}$, meanwhile, these donors with only one kidney left will live like a normal person with the same death rate if they get the proper care, so,

Above all,

$$
X_{4}=k X_{1}-d X_{4} .
$$

$\frac{d}{d t}\left(\begin{array}{l}X_{1} \\ X_{2} \\ X_{3} \\ X_{4}\end{array}\right)=\left(\begin{array}{cccc}-a-b-h & c-a & -2 & 0 \\ h & -c-d & 2 & 0 \\ -k & -k & 0 & -\frac{1}{2} k \\ h & 0 & 0 & -d\end{array}\right)\left(\begin{array}{l}X_{1} \\ X_{2} \\ X_{3} \\ X_{4}\end{array}\right)+\left(\begin{array}{l}a \\ 0 \\ k \\ 0\end{array}\right) N$

The above equations describe our model system, and if given an initial value, the system can forecast the value of $X_{1}(t) \cdots \cdots X_{4}(t)$. Facts have proven that in some years the forecast is quite accurate.

Coming to the goal of our network model design, it should save and prolong more lives, and meanwhile should cut the waiting time for the patient as short as possible.

Let 
$J=$

$\frac{\text { the accumulative total waiting time in }\left[t_{0}, t_{f}\right]}{\text { the number of patients who get the proper kidney in }\left[t_{0}, t_{f}\right]}$,

which is our index function, and apparently $\mathrm{J} \geq 0$, and the smaller $\mathrm{J}$ is, the better.

Then we will calculate the expression of $\mathrm{J}$.

$$
\begin{aligned}
& \text { Numerator }=\int_{t_{0}}^{t_{f}} X_{1}^{\prime}(t)\left(t_{f}-t\right) d t+X_{1}\left(t_{0}\right)\left(t_{f}-t_{0}\right) \\
= & \int_{t_{0}}^{t_{f}}\left(t_{f}-t\right) d X_{1}(t)+X_{1}\left(t_{0}\right)\left(t_{f}-t_{0}\right) \\
= & X_{1}(t)\left(t_{f}-t\right) \mid \begin{array}{l}
\mathrm{t}=\mathrm{t}_{\mathrm{f}} \\
\mathrm{t}=\mathrm{t}_{0}
\end{array} \int_{\mathrm{t}_{0}}^{\mathrm{t}_{\mathrm{f}}} X_{1}\left(t_{0}\right) d t+X_{1}\left(t_{0}\right)\left(t_{f}-t_{0}\right) \\
= & \int_{\mathrm{t}_{0}}^{\mathrm{t}_{\mathrm{f}}} X_{1}\left(t_{0}\right) d t
\end{aligned}
$$$$
\text { Denominator }=\int_{t_{0}}^{t_{f}}\left(2 X_{3}+h X_{1}\right) d t,
$$

So,

$$
J=\frac{\text { Numerator }}{\text { Denomin ator }}=\frac{\int_{t_{0}}^{t_{f}} X_{1} d t}{2 \int_{t_{0}}^{t_{f}} X_{3} d t+h \int_{t_{0}}^{t_{f}} X_{1} d t}=\frac{1}{\frac{2 \int_{t_{0}}^{t_{f}} X_{3} d t}{\int_{t_{0}}^{t_{f}} X_{1} d t}+h}
$$

Then, we will solve the (1), and analyze the extremum of $\mathrm{J}$ with the result.

Let $\quad \overrightarrow{\mathrm{X}}=\left(\mathrm{X}_{1} \cdots \cdots \mathrm{X}_{4}\right)^{\mathrm{T}}, \quad \vec{\alpha}=(\mathrm{a}, 0, \mathrm{k}, 0)^{\mathrm{T}}$,

$$
A_{0}=\left(\begin{array}{cccc}
-a-b-h & c-a & -2 & 0 \\
h & -c-d & 2 & 0 \\
-k & -k & 0 & -\frac{1}{2} k \\
h & 0 & 0 & -d
\end{array}\right) .
$$

Then the (1) becomes to be

$$
\frac{d \overrightarrow{\mathrm{X}}}{d t}=\mathrm{A} \overrightarrow{\mathrm{X}}+\vec{\alpha} \tilde{\mathrm{N}}
$$

Because $X_{n}=\mathrm{x}_{\mathrm{i}} \mathrm{N}(\mathrm{i}=1,2,3,4)$, let $\overrightarrow{\mathrm{x}}=\left(\mathrm{x}_{1}, \mathrm{x}_{2}, \mathrm{x}_{3}\right.$, $\left.\mathrm{X}_{4}\right)^{T}$, then

$$
\frac{d \overrightarrow{\mathrm{X}}}{d t}=\frac{d}{d t}(\overrightarrow{\mathrm{x}} \mathrm{N})=\mathrm{N}\left(\frac{d}{d t} \overrightarrow{\mathrm{x}}\right)+\overrightarrow{\mathrm{x}} \frac{d}{d t} \mathrm{~N} .
$$

So the (3) will be

$$
\begin{gathered}
\mathrm{N} \dot{\overrightarrow{\mathrm{x}}}+\overrightarrow{\mathrm{x}} \dot{\mathrm{N}}=\left(\mathrm{A}_{0} \overrightarrow{\mathrm{x}}+\vec{\alpha}\right) \mathrm{N} \\
\dot{\overrightarrow{\mathrm{x}}}=\mathrm{A}_{0} \overrightarrow{\mathrm{x}}+\vec{\alpha}-\frac{\dot{N}}{N} \overrightarrow{\mathrm{x}} \\
\dot{\overrightarrow{\mathrm{x}}}=\left(\mathrm{A}_{0}-\mathrm{I}_{4} \frac{d}{d t} \ln \mathrm{N}\right) \overrightarrow{\mathrm{x}}+\vec{\alpha} .
\end{gathered}
$$

Let $A=A_{0}-I_{4} \frac{d}{d t} \ln N$, we notice that the percentage of patients in the total population $N(t)$ is very small, so in the situation that $t_{f}-t_{0}$ is not large, such as $t_{f}-t=1$, we can apply the classic Malthusian Population Model to describe $\mathrm{N}(\mathrm{t})$, and get $N(t) \approx N_{0} e^{\mu\left(t-t_{0}\right)}$, so $\frac{d}{d t} \ln N \approx$ $\mu=$ const , and then, $A \approx A_{0}-\mu I_{4}$.

Based on the above process, the solution of the original equation can be calculated by the following formula,

$$
\vec{x}(t)=e^{A\left(t-t_{0}\right)} C_{1}-A^{-1} \vec{\alpha},
$$

where $e^{A\left(t-t_{0}\right)}=\sum_{n=0}^{\infty} \frac{1}{n !} A^{n}\left(t-t_{0}\right)^{n}$, and $C_{1} \in \mathrm{R}^{4}$ is a regular vector to be determined.

If given the initial condition

$$
\vec{x}\left(t_{0}\right)=\vec{x}_{0}=\vec{X}_{0} / N\left(t_{0}\right),
$$

We can determine

$$
\begin{aligned}
& C_{1}=\vec{x}_{0}+A^{-1} \alpha=\vec{X}_{0} / N\left(t_{0}\right)+A^{-1} \vec{\alpha}, \text { so } \\
& \vec{x}(t)=e^{A\left(t-t_{0}\right)}\left(\vec{x}_{0}+A^{-1} \alpha\right)-A^{-1} \vec{\alpha}, \quad t \in\left[t_{0}, t_{f}\right] .
\end{aligned}
$$

And with $\vec{x}(t)=\vec{x}(t) N(t)$, we can expediently calculate the value of $\vec{X}(t)$.

Substituting into $\mathrm{J}$ with the expression of $\vec{X}$, it has become the function of A. Considering a, b, c, d, h, k, which have be defined, we can get that the death percentage of the patients in the waiting list, $b$, closely relate to the average length of waiting time. If we apply Poisson distribution to describe the renal allograft dysfunction, so c approximately equals the reciprocal of the average length of life after transplant, which is related to requirements on the kidney medical matching before distribution. The more strict requirements on the kidney medical matching are, the longer the expected total length of life of transplants. But $a$ and $d$ nearly are effected by our kidney distribution policies because they will just effect only very few patients' life length, while $h$ and $k$ reflect the psychological dynamics of the people, which will not be directly changed by our distribution policies. So we should take $b$ and $c$ as controllable variables, while search $a, d, h, k$ from the public data. 
A natural idea is to let $b$ and $c$ as small as possible. In the actual operation, to decrease the average waiting time, we have to loose the condition, while the average waiting time will become longer, if we strict the requirements on matching much more, so our goal is to get the best combination of $b$ and $c$.

Because (1) is resolvable, and the form of the solution ensures it is continuous derivative about $t$, so we substitute $\vec{X}$ into it, and we can get the expressions of $J(b, c)$. In the actual work, this calculation is very complex, but thanks to some powerful symbol computing mathematical software, it will be quickly done.

From the results, we come to the conclusion that $J(b, c)$ is continuous derivative about (b,c), and apparently $0 \leqslant b \leqslant 1,0 \leqslant c \leqslant 1$. So it's sure to get the minimum of $J(b, c)$. Theoretically speaking, we can apply

$$
\left\{\begin{array}{l}
\frac{\partial}{\partial b} J=0 \\
\frac{\partial}{\partial c} J=0
\end{array}\right.
$$

to get the extremum from the solutions, and then screen out. But in the actual work, we can apply numerical methods to get the numerical solution, and even to analyze the trend of $\mathrm{J}$ by drawing the $3 \mathrm{D}$ imagines to guide policies.

\subsection{Testing and Result Analysis}

After the analysis of data (2004-2005) in America, we get the figure (Figure 4) about $\mathrm{J}(\mathrm{b}, \mathrm{c})$ as follows.
We find that if $\mathrm{c}$ is given, $\mathrm{J}$ changes with $\mathrm{b}$ steadily, while when c increases, J decreases rapidly. So the bottleneck of the current situation in America is c, that is, the rate of renal allograft dysfunction. Based on these analyses, we recommend that benefit the patients with higher matching rate, and reduce the weight of waiting time to let $\mathrm{c}$ as small as possible.

Coming to the details, according to actual factors, you should determine the domain of $\mathrm{J}(\mathrm{b}, \mathrm{c})$, and then find the nearest point $\left(b_{0}, c_{0}\right)$ to axis $\mathrm{c}=0$ in this area. If there are many such points, you should take the smallest value of $\mathrm{b}$ as $b_{0}$.

For example, considering

$$
\left\{\begin{array}{l}
\frac{2}{b}+\frac{1}{c} \leq 40 \\
0.05 \leq c \leq 0.8 \\
0.01 \leq b \leq 0.2
\end{array}\right.
$$

based on the above rules, we should take $\left(b_{0}, c_{0}\right)=$ $(0.1,0.05)$ as data to assist guidance. You should adjust next year' distribution policy, when you find $(b, c)$ doesn't accord last year's statistical data. $b<b_{0}$ and $c<c_{0}$ will not appear because $\frac{2}{b}+\frac{1}{c}=40$. If $b>b_{0}$, you should increase the weight of waiting time in the next year's evaluation policy; if $c>c_{0}$, you should increase the weight of matching rate.

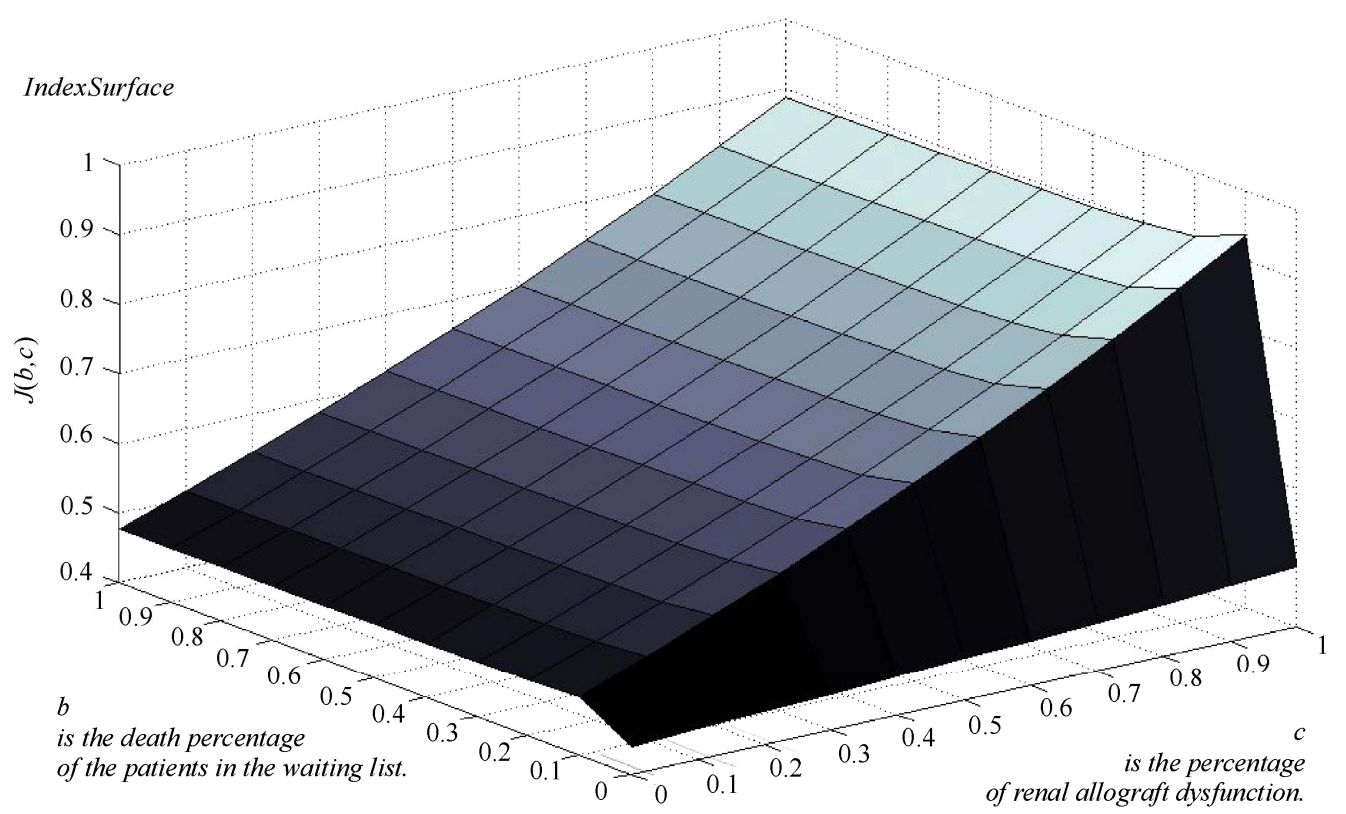

Figure 4. $\mathrm{J}(\mathrm{b}, \mathrm{c})$ situation analysis. 


\subsection{Conclusions and Solutions}

When distributing kidneys, we are always tending to hesitate on balancing waiting time and the matching rate. Let's take a case for example. A kidney matches a patient completely who just now entered the waiting list, while it is barely compatible for a patient who has waited for a year. Then the doctor will be puzzled how to distribute this precious kidney. By using our model, we could get the average latency time and the average matching rate. If in the average waiting time, we should consider the average matching rate firstly and distribute the kidney to the patient with the best match, while out of the average waiting time, we should distribute the kidney to the patient with the longest waiting time.

The potential bottleneck is the contradiction between matching rate and waiting time. We will evaluate the latency risks during the waiting time in III-A.

It is hard to say that the network divided into smaller ones is good or not, because the situation varies from state to state. In some areas the kidney resource is very rare, while the situation is opposite in the other areas. Establishing a large network could balance resources in all fields. Of course, the patients must have an operation immediately because the warranty for a cadaver kidney is only 1-2 days [12]. For the reason of distance, it is more appropriate to choose a donor in a nearer area. Anyhow, it is feasible to substitute the data of the district into our model and evaluate overall.

The ways to save and prolong more lives can be reflected by parameters $b, c, h$ which are mentioned in our model. In our distribution network, the index function is

$J=$

$\frac{\text { the accumulative total waiting time at }\left[t_{0}, t_{f}\right]}{\text { the number of patients who get the proper kidney at }\left[t_{0}, t_{f}\right]}$

We can change it to be $J=$ the number of patients who get the proper kidney. So we can decrease the restrictions to pursue the one and only target, saving and prolonging more lives, and the system will be more effective.

\section{DISTRIBUTION DECISION TREE}

\subsection{Analysis of the Problem}

As the number of organs for transplanting is restricted, while a large number of patients need them, we have to consider how to distribute these organs fairly, reasonably and effectively.

The typical approach for distribute is linear matching score ( score $\left.=\sum_{i=1}^{n} w_{i} a_{i}+b\right)$, but we believe there are several defects in this method.

1) Variables are difficult to normalize. For example,
$0<$ age $<100$, commonly, as there are $\mathrm{A}, \mathrm{B}, \mathrm{AB}$ and $\mathrm{O}$ four blood types. How to put them into a uniform and comparable range?

2) The weight for each variable is hard to confirm.

So we introduce the decision tree to get across this morass. A decision tree is flow-chart-like tree structure, where each internal node denotes a test on an attribute, each branch represents an outcome of the test, and leaf nodes represent classes or class distributions.

\subsection{Attributes for Decision}

We mainly consider these attributes while distributing:
a. Transplant centre effects
b. HLA match rate
c. Incompatibility rate
d. Patient renal disease
e. Waiting time

Each of them has characteristic itself. Based on these characteristics, we classify them as follows.

Boolean Attribute: Transplant centre effects and $\mathrm{Pa}-$ tient renal disease. Kidney transplanting should be operated as soon as possibly. If the distance between the kidney and the patient is too far, it will be impossible to make this operation. Based on this factor, patients should be classified into two parts, incompatibility and compatibility. If the patient is suffering other disease, e.g. AIDS or cancer, our advice is that they are not fit to distribute a kidney.

Continuous Attribute: HLA match rate and Incompatibility rate。Based on the number of match points, the output results will be divided into many classes, such as $\mathrm{r}>90 \%, 90 \%>\mathrm{r}>60 \%$, and $\mathrm{r}<60 \%$ or the top $10 \%$, $20 \% \sim 50 \%$, and so on. Treatment will all depend on the situation.

Consult Attribute: Waiting time. If a patient waits for a kidney for a long time, he will have a priority.

The attributes we described are classic, and there are a quantity of attributes worth to interpret, e.g. psychology, age and so on. They can also be classified as we talked about before and we will make further discussions in 3.3.

\subsection{Description of Decision Tree}

The basic algorithm for decision tree induction is a greedy algorithm that constructs decision trees in a topdown recursive divide-and-conquer manner. The basic strategy is as follows.

The tree starts as a single node representing the information of patients which maybe in large scale and the only kidney for distribute.

A branch is created for each known value of the test attribute, and the patients are partitioned accordingly.

Finally, every patient are labelled by a leaf which representing the match rate of the patient and the kidney, in 


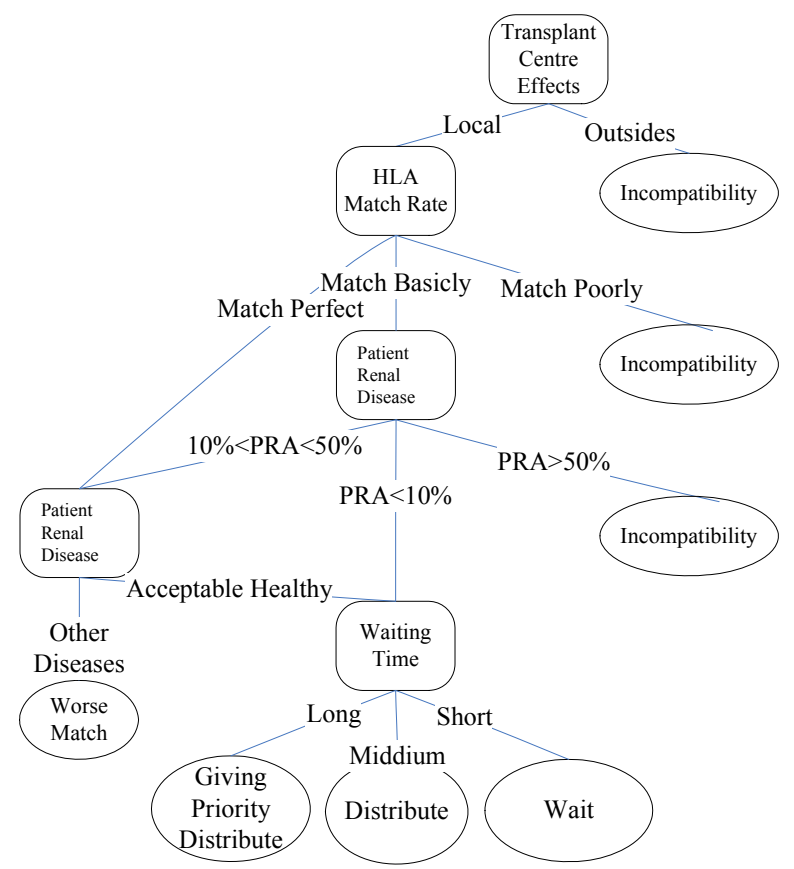

Figure 5. Basic decision tree to make the distribution.

fact, we can consider that they are classified. The patient who gets the best match rate will receive this kidney.

The implementation and other interpreters of decision tree, you may refer to ID3 [8], C4.5 [6] and CART[7].

\subsection{Improvement}

In this issue, we talk about the improvement be made on the tree when considering another attribute, e.g. ABO blood type.

First, ascertain which class this attribute should be classified. ABO blood type is Boolean Attribute.

$\mathrm{ABO}$ blood type is a very important attribute, it is suited be treated as a child of Transplant centre effects and a parent of HLA match rate.

Since other part of the tree is needless to modify, we improve the tree, as in Figure 6.

\subsection{Conclusions}

This solution can be compared and evaluated according to the following criteria:

Distribution accuracy: This refers to the ability of the model to correctly predict the class label of new or previously unseen data. In this model, every patient can receive an excellent estimate. (n).

Speed: Time cost of operations on the tree mostly is $\mathrm{O}$

Robustness: If the condition of a patient is very special. We can consider his attribute and improve the tree.

Scalability: The efficiency of existing decision tree algorithms, such as ID3 and C4.5, has been well established for relatively small data sets. Algorithms for the

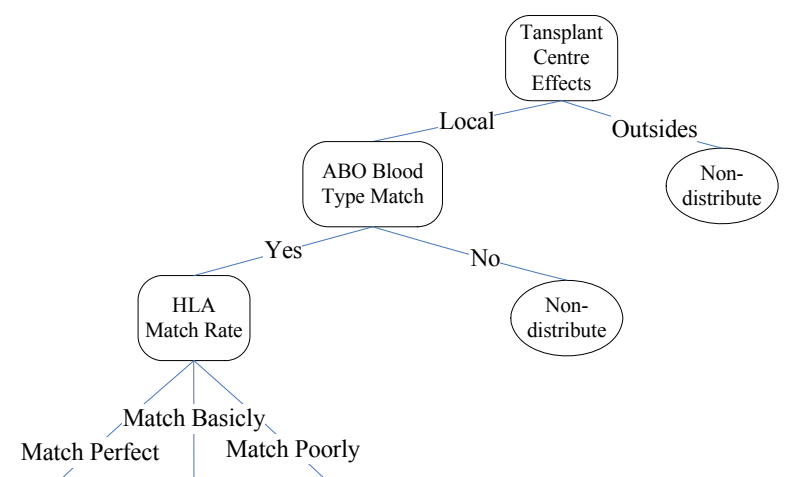

Figure 6. Advanced decision tree to make the distribution.

induction of decision trees from very large training sets include SLIQ and SPRINT.

\section{PROCEDURES TO MAXIMIZE THE NUMBER AND QUALITY OF EXCHANGES}

\subsection{Analysis of the Problem}

There are a number of patients and every patient has a willing donor. Some pairs are compatible, while the others are afflictive because of blood-type incompatibility or poor HLA match and so on. For the compatible pairs, the transplants are convenient, while the opposite ones are helpless even though the donor is self-giving for his partner. "This is a significant loss to the donor population and worthy of consideration when making new policies and procedures." (http://www.optn.org/ latest Data/ view DataReports.asp)

This situation may be improved by a kidney exchange system, which can take place either with a living donor or in the cadaver queue. Our goal in this part is to devise a procedure that more patients receive more compatible kidneys.

\subsection{Assumptions}

1) One pair is a patient with a donor.

2) We focus on the matching rate which is based on the blood-type compatibility and HLA match.

3) As for psychological dynamics, we assume that all donors who take part in the exchange system are so altruistic that they are willing to contribute their kidneys to any patient in the system.

4) Every patient is healthy enough that he doesn't need transplant immediately, that is, he has enough waiting time, while every donor is healthy and willing to donate any time.

\subsection{Definitions and Key Terms}

$N$ is the number of pairs. 
$x_{i j} \quad x_{i j} \in\{1,0\} \quad$ where $1 \leq \mathrm{i} \in \mathrm{N}, 1 \leq \mathrm{j} \leq \mathrm{N}, \mathrm{i}, \mathrm{j} \in$

Natural Number. If the patient in Pair i receives a kidney from Pair j,$x_{i j}=1$, otherwise, $x_{i j}=0$.

$r_{i j} \quad r_{i j} \in[0 \%, 100 \%]$, where $1 \leq \mathrm{i} \in \mathrm{N}, 1 \leq \mathrm{j} \leq \mathrm{N}, \mathrm{i}, \mathrm{j} \in$

Natural Number. It is the match rate of the patient from Pair $\mathrm{i}$ with the kidney from Pair $\mathrm{j}$.

$X \quad$ is the Matrix whose elements are $X_{i j}$.

$R \quad$ is the Matrix whose elements are $r_{i j}$.

\subsection{Design of the Model}

We build an Integer Programming model to determine how to distribute all the kidneys to the appropriate patients. We have two kinds of best targets, which can be applied in different situation:

1) The entity matching rate is maximal

2) The number of successful exchange pairs is maximal

First, Fill Matrix R using decision tree which we described in session 3 .

MODEL:

Objective function:

$$
\max \text { result }=R X^{T}
$$

Constraints:

$$
\begin{aligned}
& \sum_{i=1}^{n} X_{i k}=1 ; \mathrm{k}=1,2 \ldots \mathrm{n} \\
& \sum_{j=1}^{n} X_{l j}=1 ; 1=1,2 \ldots \mathrm{n}
\end{aligned}
$$

where (5) means one kidney can be only distributed to one patient, while (6) means one patient only gets one kidney.

This model is a classical Integer Programming which can be solved by Lingo etc.

Based on the model, we establish our algorithm:

Step 1. Establish the database of all pairs, including the blood types, HLA characters, and other personal data like age, health situation etc.

Step 2. Fill the Matrix $R$ through decision tree (as we provide, or established by users themselves), referring to session 3.

Step 3. Solve the model, and gain a nice exchange scheme.

\subsection{Testing and Sensitivity Analysis}

In order to illuminate how the procedure works, we simulate a representative example.

We try our best to make sure that the data is random, in order to make it universal. The Matrix R produced by Matlab7.1 is as follows,

$\left.\begin{array}{cccccccccc}\mathrm{R}=[63 & 0 & 68 & 0 & 0 & 93 & 0 & 0 & 77 & 62 \\ 82 & 0 & 0 & 0 & 0 & 0 & 89 & 70 & 79 & 76 \\ 0 & 0 & 0 & 0 & 0 & 86 & 0 & 0 & 89 & 0 \\ 0 & 0 & 77 & 0 & 58 & 68 & 67 & 100 & 0 & 94 \\ 57 & 0 & 0 & 0 & 0 & 0 & 97 & 0 & 78 & 0 \\ 0 & 75 & 0 & 0 & 74 & 0 & 78 & 89 & 93 & 100 \\ 0 & 61 & 0 & 89 & 0 & 67 & 0 & 0 & 0 & 0 \\ 60 & 0 & 0 & 0 & 0 & 72 & 78 & 0 & 0 & 61 \\ 90 & 0 & 67 & 63 & 0 & 83 & 0 & 65 & 0 & 60 \\ 60 & 0 & 0 & 0 & 66 & 0 & 0 & 75 & 0 & 0\end{array}\right] ;$

We solve the problem by Lingo 8.0, and the result is,

Objective value: $\mathrm{w}=822.0000$

$\begin{array}{lcc}\mathrm{X}(1,3) & 1.000000 & 68.00000 \\ \mathrm{X}(2,10) & 1.000000 & 76.00000 \\ \mathrm{X}(3,9) & 1.000000 & 89.00000 \\ \mathrm{X}(4,8) & 1.000000 & 100.0000 \\ \mathrm{X}(5,7) & 1.000000 & 97.00000 \\ \mathrm{X}(6,2) & 1.000000 & 75.00000 \\ \mathrm{X}(7,4) & 1.000000 & 89.00000 \\ \mathrm{X}(8,6) & 1.000000 & 72.00000 \\ \mathrm{X}(9,1) & 1.000000 & 90.00000 \\ \mathrm{X}(10,5) & 1.000000 & 66.00000\end{array}$

In Figure 7, the arrow shows a kidney is passed from pair $\mathrm{j}$ to pair $\mathrm{i}$.

In this example, if we had not applied this exchange system, only one pair can accomplish the transplant, that is, Pair 1 whose matching rate is 63 . However, through taking part in the exchange system, all the pairs obtain the gratifying kidney. This weight matrix can be considered to be consummate, but then, there are situations that some pairs can not obtain appropriate kidneys even though they donate kidneys to others. With the quit of the satisfied pairs and entry of new pairs, this network is

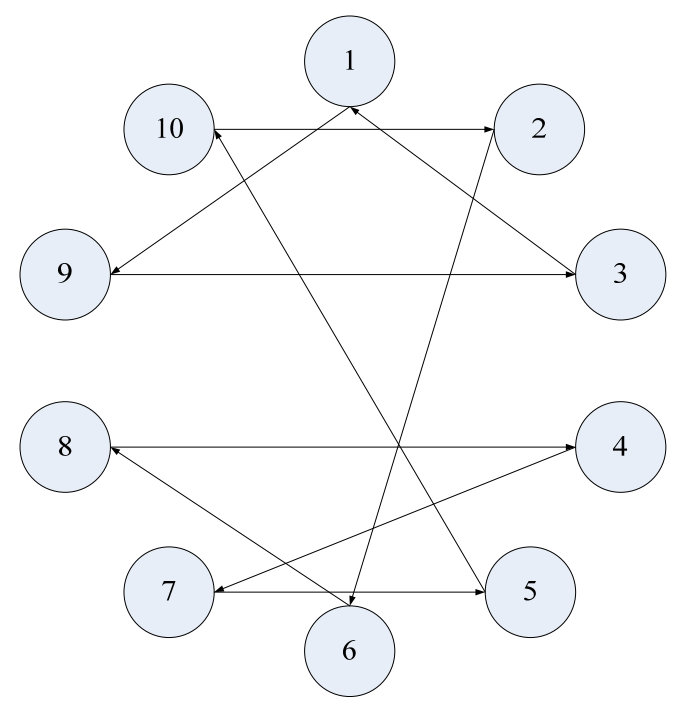

Figure 7. An example of kidney exchange system. dynamic, and in the next distribution, these patients will 
have the priority to get donor or cadaver kidneys.

\subsection{Justification}

Because we apply Integer Programming, so the solution maybe is the best, and we take the pairs in the system fairly, so the matching process and result is meaningful for the current exchange system.

\subsection{Conclusions}

In this model, all pairs who would like to exchange their organs register at first, and then the system collects the information which is useful in the organ matching. Following the procedure that is proposed in 4.4 , we can get the data that how many more annual transplants will generate.

Because of the matching problem, maybe some of the patients can't get kidneys even though they have provided kidneys for others. But to advocate donors, as compensation these people will rank a higher priority in the waiting list, while patients in the current waiting list have a willing but incompatible donor will benefit for this exchange system, and you will get the number of the more annual transplants through the procedure we proposed.

\section{STRATEGIES FOR THE PATIENT TO MAKE A BETTER DECISION}

\subsection{Analysis of the Problem}

A patient maybe always face a hard and awkward situation and need a strategy to decide whether to take an offered kidney, wait for a better match from the cadaver queue or to participate in a kidney exchange. We propose a strategy considering the risks, alternatives, and probabilities to help patients to make a better decision and come to a better result.

When facing the situation that whether to take a barely compatible kidney, the patient should consider and evaluate the risk of waiting, the effect of transplant, and the financial factors to make a decision. After study a mount of relative materials, we find out that there are different exchange modes in different countries. To be clear, we take a popular mode as an example to study and discuss, which is, once a patient decides to participate in a kidney exchange, and put out an offer, then he will get a very authorized promise that he will get a better matching kidney in a given certain time.

\subsection{Assumptions and Definitions}

$\xi$ is the left life length of the patient.

$\eta$ is the interval length between two offers(last offer he gave up, and this offer he is waiting for) for the patient.

$\zeta$ is the normal life time length of the transplanted kidney.

$\xi, \eta$, and $\zeta$ all obey exponential distribution, and their parameters are expectable, and $\xi, \eta$, and $\zeta$ are statistical independent. The parameters of $\zeta, \lambda_{\zeta}$, are relative to matching rate, the health condition of the patients and the donors and the donors kidneys. Let $\lambda_{\zeta}$ is directly proportional to the matching rate, that is, after a patient took a cadaver kidney, $\lambda_{\zeta}=r^{-1} \lambda_{\zeta}^{(0)}$, where $r \in(0,1]$ is the matching rate. $\lambda_{\zeta}^{(0)}$ is a const determined by the condition of the patient. Under the same condition, the living donors let $\lambda_{\zeta}=\frac{1}{2} r^{-1} \lambda_{\zeta}^{(0)}$.

Our goal is to design the best strategy for the patient when he faces the situation that whether he should take the offer. So we don't consider the death caused by other factors other than nephropathy. For a certain patient, $r$, a random variable, obeys uniform distribution on $\left[r_{0}, 1\right]$. (Other distributions can be solved by applying their inverse functions, which refer to Angel R. Martinez and Wendy L. Martinez).

$r_{0}$ is determined by the distribution policies in the network.(e.g. some network only distributes kidney to the patient with the matching rate higher than $50 \%$, so $r_{0}=0.6$ ). Because the probability of the death caused by the transplant operating is very small and minimally different for different patients, so we assume the patients will not die on the operating table.

\subsection{Design of the Model}

Let $L=p$ \{transplant success $\} E \zeta$, because during the waiting time, the patients will face the nephropathy death risk, and after successful transplant, before the renal allograft dysfunctions, the patients will enjoy the normal life, so $L$ characterizes the expectation of the patient after making a decision, which is our index function.

Our goal is to propose a strategy for the patients to let $L$ maximum. And the alternative programs are:

Strategy 1. Take the first offer without hesitation, and the matching rate is $r_{f}$.

Strategy 2. Always refuse the offers whose matching rate is lower than $r_{e}$, until get the kidney with $r \geq r_{e}$.

Strategy 3. Participate in a kidney exchange, and exchange the barely compatible kidney with our needy patients.

The details of the model are as follows.

Take the first offer without hesitation.

Based on assumption 5 ( II ,B),

$P\{$ successful transplant $\}=1$ 
So,

$$
L_{1}=1 E \zeta=\lambda_{\zeta}^{-1}=r_{f}\left(\lambda_{\zeta}^{(0)}\right)^{-1}
$$

Wait for a $r \geq r_{e}$ match from the cadaver queue.

$P\{$ successful transplant $\}$

$=P\{$ the cadaver kidney arrives before the patient dies $\}$

$=\sum_{n=1}^{\infty} P\left\{\right.$ the $n^{\text {th }}$ offer arrives before the patient dies $\}$

$* P\left\{\right.$ the first time that the $n^{\text {th }}$ offer satisfies $\left.r \geq r_{e}\right\}$

$=\sum_{n=1}^{\infty} P\left\{\xi>\eta_{n}\right\} *\left(\frac{r_{e}-r_{0}}{1-r_{0}}\right) \frac{1-r_{e}}{1-r_{0}}$.

Because $\eta_{n}$ is independent identical distribution with $\eta . \xi$ and $\eta$ are statistical independent, so

$$
\begin{aligned}
& P\{\text { successful transplant }\} \\
& =P\{\xi>\eta\} \\
& =\iint_{0<y<x} \lambda_{\xi} e^{-\lambda_{\xi} x} \lambda_{\eta} e^{-\lambda_{\eta} y} d x d y \\
& =\int_{0}^{+\infty} d x \int_{0}^{x} \lambda_{\xi} \lambda_{\eta} \operatorname{EXP}\left\{-\lambda_{\xi} x-\lambda_{\eta} y\right\} d y \\
& =\frac{\lambda_{\eta}}{\lambda_{\xi}+\lambda_{\eta}} .
\end{aligned}
$$

So,

$$
\begin{aligned}
& L_{2}=\frac{\lambda_{\eta}}{\lambda_{\xi}+\lambda_{\eta}} * E \zeta \\
& =\frac{\lambda_{\eta}}{\lambda_{\xi}+\lambda_{\eta}}\left(\lambda_{\xi}\right)^{-1} \\
& =\frac{\left(1+r_{e}\right) \lambda_{\eta}}{2\left(\lambda_{\xi}+\lambda_{\eta}\right)}\left(\lambda_{\xi}^{(0)}\right)^{-1}
\end{aligned}
$$

Participate in a kidney exchange.

The waiting time for the next offer obeys truncated exponential distribution, and let $T$ the longest time to get the promised kidney, so

$$
\begin{aligned}
& F_{\eta}(t)=P\{\eta<t\}
\end{aligned}
$$

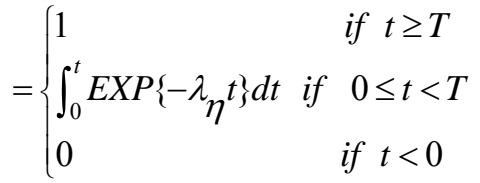

And then,

$P\{$ successful transplant $\}=P\{\eta<\xi\}$

$$
\begin{aligned}
& =\iint_{0<y<x} \lambda_{\xi} e^{-\lambda_{\xi} x} d x d F_{\eta}(y) \\
& =\int_{0}^{+\infty} \lambda_{\xi} e^{-\lambda_{\xi} x}\left(F_{\eta}(x)-F_{\eta}(0)\right) d x \\
& =\int_{0}^{T} \lambda_{\xi} e^{-\lambda_{\xi} x}\left(\int_{0}^{x} \lambda_{\eta} e^{-\lambda_{\eta} y} d y\right) d x+\int_{T}^{+\infty} \lambda_{\xi} e^{-\lambda_{\xi} x} d x \\
& =\int_{0}^{T} \lambda_{\xi} e^{-\lambda_{\xi} x}\left(1-e^{-\lambda_{\eta} x}\right) d x+\int_{T}^{+\infty} \lambda_{\xi} e^{-\lambda_{\xi} x} d x \\
& =1-\int_{0}^{T} \lambda_{\xi} e^{-\left(\lambda_{\xi}+\lambda_{\eta}\right) x} d x \\
& =1-\frac{\lambda_{\xi}}{\lambda_{\xi}+\lambda_{\eta}}\left(1-e^{-\left(\lambda_{\xi}+\lambda_{\eta}\right) T}\right) \\
& =\frac{\lambda_{\eta}}{\lambda_{\xi}+\lambda_{\eta}}+\frac{\lambda_{\xi}}{\lambda_{\xi}+\lambda_{\eta}} e^{-\left(\lambda_{\xi}+\lambda_{\eta}\right) T} .
\end{aligned}
$$

So,

$L_{3}=P\{$ successful transplant $\} * E \zeta$

$=P\{$ successful transplant $\} \lambda_{\xi}^{-1}$

$=P\{$ successful transplant $\} 2\left(1+r_{f}\right) \frac{1}{2}\left(\lambda_{\xi}^{(0)}\right)^{-1}$

$$
=\left(1+r_{f}\right)\left[\frac{\lambda_{\eta}}{\lambda_{\xi}+\lambda_{\eta}}+\frac{\lambda_{\xi}}{\lambda_{\xi}+\lambda_{\eta}} e^{-\left(\lambda_{\xi}+\lambda_{\eta}\right) T}\right]\left(\lambda_{\xi}^{(0)}\right)^{-1} a .
$$

\subsection{Testing and Sensitivity Analysis}

If we want to compare $L_{1}$ and $L_{3}$, just need to know the values of $r_{f}, T, \lambda_{\xi}, \lambda_{\eta}$, and $r_{f}, T$ are all already known. Because $\eta$ and $\xi$ all obey exponential distribution, so $E \xi=\lambda_{\xi}^{-1}, E \eta=\lambda_{\eta}^{-1}$, and then $\lambda_{\xi}=(E \xi)^{-1}$, $\lambda_{\eta}=(E \eta)^{-1}$.

Because $E_{\xi}$ is the expected left life length of the patient without transplant, while $E_{\eta}$ is the average waiting time length for the next kidney, which can all be estimated from the public data.

For example, in a country, the average lasting time for a patient without transplant is 10 years, and the average waiting time for a patient to get transplant is 5 years, so $\lambda_{\xi}=0.1, \lambda_{\eta}=0.2$.

$$
\begin{aligned}
& \text { If } r_{f}=60 \%, T=5, \text { then } \\
& L_{1}=60 \% \cdot \frac{0.2}{0.1+0.2} \cdot\left(\lambda_{\xi}^{(0)}\right)^{-1}=\frac{2}{5}\left(\lambda_{\xi}^{(0)}\right)^{-1}=0.4\left(\lambda_{\xi}^{(0)}\right)^{-1} \\
& L_{3}=(1+60 \%) \cdot\left[\frac{0.2}{0.1+0.2}+\frac{0.1}{0.1+0.2} \cdot e^{-1.5}\right] \cdot\left(\lambda_{\xi}^{(0)}\right)^{-1} \\
& \approx 1.1857\left(\lambda_{\xi}^{(0)}\right)^{-1}
\end{aligned}
$$

So in this situation, the patient should take part in ex- 
change.

From the above process, we can come to the conclusion that, the larger $\lambda_{\xi}, r_{f}$ and $\mathrm{T}$ are, the smaller $\lambda_{\eta}$ is, and then the strategy should be taking the offer right now; the smaller $\lambda_{\xi}, r_{f}$ and $\mathrm{T}$ are, the larger $\lambda_{\eta}$ is, then the strategy should be taking part in exchange and waiting for a better kidney.

\subsection{Strength and Weakness}

Based on our assumptions, the distribution of any event a patient may face is clear, that is, we can expediently calculate the expected values, variances and so on. So we have conditions to design different index functions.

We tried the following index functions,

$K_{i}=E\{$ the left life time of the patients

who are making the decision

| at most transplant kidneys two times, choose the $i^{\text {th }}$ strategy

$i=1,2,3$

After analyze the decision tree, we can get the expressions of $K_{1}, K_{2}$, and $K_{3}$, and easily solve them. But finally we discard it, because of the following analysis. The average life length of transplant kidneys is almost 10 years, we can not forecast what will happen in this 10 year, such as the death caused by other factors, and the man-made kidney technology progress rapidly, so it's not meaningful to make a 20 -years forecast ( transplant kidneys two times).

$$
\begin{aligned}
& C_{i}=E \text { \{the expense before } \\
& \text { the first transplant }, i=1,2,3 \\
& \text { |choose the } i^{\text {th }} \text { strategy\} }
\end{aligned}
$$

Because $\mathrm{C}$ increases with the expected waiting time for the first transplant, and for the same person, the proportion coefficient is the same, so we can let it 1 .

So

$$
\begin{aligned}
& C_{1}=0 \\
& C_{2}=E\{\min \{\xi, \eta\} \mid 2\} \\
& =\iint_{0<x<y} x d F_{\xi}(x) d F_{\eta}(y)+\iint_{0<y<x} y d F_{\xi}(x) d F_{\eta}(y) \\
& =\frac{\lambda_{\xi}}{\lambda_{\xi}+\lambda_{\eta}^{\prime}} \cdot \frac{1}{\lambda_{\xi}}+\frac{\lambda_{\eta}^{\prime}}{\lambda_{\xi}+\lambda_{\eta}^{\prime}} \frac{1}{\lambda_{\eta}^{\prime}}=\frac{2}{\lambda_{\xi}+\lambda_{\eta}^{\prime}}
\end{aligned}
$$

where $\lambda_{\eta}^{\prime}=\frac{1-r_{e}}{1-r_{0}} \lambda_{y}$, so

$$
\begin{aligned}
& C_{2}=\frac{2\left(1-r_{0}\right)}{\lambda_{\xi}\left(1-r_{0}\right)+\lambda_{\eta}\left(1-r_{e}\right)} . \\
& C_{3}=E\{\min \{\xi, \eta\} \mid 3\} \\
& =\iint_{0<x<y} x d F_{\xi}(x) d F_{\eta}(y)+\iint_{0<y<x} y d F_{\xi}(x) d F_{\eta}(y) \\
& =\int_{0}^{T} d F_{\eta}(y) \int_{0}^{y} x e^{-\lambda_{\xi} x} d x+\int_{T}^{+\infty} d F_{\xi} \int_{0}^{x} y d F_{\eta}(y)
\end{aligned}
$$

$\mathrm{C}$ can be applied as the index to evaluate strategies, which is the smaller the better.

\subsection{Conclusions}

By far we have established the model with all the expressions and equations for the strategy. After the system calculate the three results, $L_{1}, L_{2}$ and $L_{3}$, the patient just need to compare and choose the maximum.

Note: Considering $0<r_{0} \leq r_{f}<r_{e}<1, \quad \lambda_{\xi}, \lambda_{\eta}, \lambda_{\zeta}, \lambda^{(0)}$ $>0$, and $T>0$, we can get $L_{3}-L_{2}>0$.

So in the actual strategy, the patients just need to choose between "take it right now" and "take part in exchange". It is apparent and easy to explain that, receiving an offer is an opportunity, but in strategy 2 , the patient gives it up just because it's not satisfied, while in strategy 3 , this opportunity is used fully and becomes an advantage.

\section{DISCUSSIONS AND PROSPECTS}

In the donor kidney distribution, whether an individual or entity, the matching rate is very important and we should try our best to match the kidneys. While the waiting time is not necessarily the most important factor, so the patient should participate in kidney exchange, rather than get a rarely matching kidney. Because in some acceptable wait time, he has a very high probability of getting a good matching rate kidney.

In the waiting list, the patient with the payback who has given up a kidney should get the highest priority.

The illness condition of the patient is another important factor to determine the order of the patient in the waiting list. When the condition is quite serious, so the more time the patient waits, the greater the probability of his death is. For this kind of patients, it is necessary to loose the matching requirements and give them priority to get kidneys. And our system can achieve the approximate global optimal solution.

Our model can also be applied to online products distribution, the satisfaction rate of the customs is just like the matching rate in our model.

With the progress of the technology and society, maybe we should pay close attention to organ clone 
technology, organ sale legalization and the increasing single kidney crowd.

\section{ACKNOWLEDGEMENT}

The paper is supportted by 2008-China Shenzhen Inovation Technology Program (SY200806300211A).

\section{REFERENCES}

[1] A. R. Martinez and W. L. Martinez, Computational statistics handbook with MATLAB, CHAPMAN \& HALL/ CRC.

[2] A. Schrijver, (1986) Theory of linear and integer programming, Wiley-Interscience Series in Discrete Mathematics.

[3] B. B. Dunaev, (1986) Statistical model of change in population numbers, Cybernetics and Systems Analysis, 22(4), July.

[4] E. Newcomer and G. Lomow, (2004) Understanding SOA with web services (independent technology guides), Addison-Wesley Professional.

[5] Y. Hao, Y. H. Ding, G. H. Wen, (2001) SOAP protocol and its application $[\mathrm{J}]$, Computer Engineering, 27(6), $128-130$.

[6] J. R. Quinlan, (1993) C4.5: Programs for machine learning, Morgan Kaufmann Publishers Inc., March.

[7] L. Breiman, J. H. Friedman, R. A. Olshen, and C. J. Stone (1984), Classification and regression trees, Wadsworth International Group, Belmont, California.

[8] J. R. Quinlan, (1986) Induction of decision trees, Machine Learning, (1), 81-106.

[9] M. Mehta, R. Agrawal, and J. Rissanen (1996) SLIQ: A fast scalable classifier for data mining, Extending Database Technology.
[10] C. L. Li and L. Y. Li (2003) Apply agent to build service management $[\mathrm{J}]$, Journal of Network and Computer Applications, 26, 323-340.

[11] M. Keen, A. Acharya, S. Bishop, A. Hopkins, S. Milinski, C. Nott, R. Robinson, J. Adams, and P. Verschueren, (2005) Patterns: Implementing an SOA using an enterprise service bus.

[12] OPTN, SRTR. (2005) The U. S. organ procurement and transplantation network and the scientific registry of transplant recipients, 2005 OPTN / SRTR Annual Report.

[13] S. Oliver, V. B. Prasad, D. Nigel, et a1., (2003) Leveraging the grid to provide a global platform for ubiquitous computing research, Technical Report Lancaster University, $\mathrm{CSEG} / 2 / 03$ [EB/OL].

[14] Shafer, Agrawal, Mehta, (1996) SPRINT: A scalable parallel classifier for data mining [C], Proceedings of the 22nd International Conference on Very Large Data Bases Mumbai, India.

[15] H. Yamada and T. Kasvand, (1986) DP matching method for recognition of occluded, reflective and transparent objects with unconstrained background and illumination, in Proc. 8th Int. Conf. Pattern Recog., Paris, 95-98.

[16] E. Newcomer and G. Lomow, (2005) Understanding SOA with web services, Addison Wesley, ISBN 0-32118086-0.

[17] M. Bell, (2008) Introduction to service-oriented modeling, service-oriented modeling: service analysis, design, and architecture, Wiley \& Sons, 3, ISBN 978-0-47014111-3.

[18] T. Erl, (2005) Service-oriented architecture: Concepts, technology, and design, Upper Saddle River: Prentice Hall PTR, ISBN 0-13-185858-0. 